\title{
Polyamine Metabolism and Obesity: Polyamine Metabolic Enzymes Involved in Obesity
}

\author{
Nihal Büyükuslu ${ }^{1 *}$, Rabia İclal Öztürk \\ ${ }^{1}$ Department of Nutrition and Dietetics, School of Health Sciences, Istanbul Medipol University, Istanbul, Turkey
}

\begin{abstract}
The natural polyamines, putrescine, spermidine and spermine are distributed widely in all cells including adipocytes. They are involved in several physiological processes involving gene expression and cell proliferation. The body pool of polyamines is maintained by endogenous biosynthesis, intestinal microorganisms and the diet. A correlation between fat metabolism and polyamine metabolism has been reported in several studies. It was shown that the inhibition of polyamine metabolism enzymes had been associated with increased adipose tissue and weight gain in human and animal models. Ornithine decarboxylase (ODC) and S-adenosylmethionine decarboxylase (AdoMetDC) are anabolic enzymes; and spermidine/ spermine N1-acetyltransferase (SSAT) and polyamine oxidase (PAO) are catabolic enzymes which regulate polyamine homoeostasis. Genetically altered polyamine metabolic enzymes resulted in higher tissue adipose content and weight gain indicating potential links between obesity and polyamine metabolism. This review aims to provide details on previously reported sources of data published on polyamine metabolism and obesity.
\end{abstract}

Key words: Polyamine, obesity, ornithine decarboxylase, S-adenosylmethionine decarboxylase, spermidine/spermine N1-acetyltransferase, polyamine oxidase

\section{INTRODUCTION}

Putrescine, spermidine and spermine are the natural amines distributed widely in all cells including adipocytes. They are involved in several physiological processes such as DNA stabilization, regulation of gene expression, ion channel function and cell proliferation. ${ }^{1}$ The aim of this review is to evaluate the relationship between polyamine metabolic enzymes and lipid metabolism to shed light on the treatment of obesity.

*Corresponding author: Nihal Büyükuslu, e-mail: nbuyukuslu@medipol.edu.tr (Received 06 March 2018, accepted 28 March 2018) 


\section{Polyamines and metabolism}

Polyamine homoeostasis is regulated by synthesis, degradation and membrane transport of polyamines in mammalian cells (Figure 1). ${ }^{2}$ Ornithine decarboxylase (ODC) and S-adenosyl-L-methionine decarboxylase (AdoMetDC) are the two key enzymes in the polyamine biosynthetic pathway. They are strongly regulated by feedback mechanisms at transcriptional, translational and posttranslational levels. Spermidine/spermine N1-acetyltransferase (SSAT) and polyamine oxidase (PAO) control the catabolism of polyamines in the polyamine metabolism. An increase in cellular polyamines rapidly induces SSAT, resulting in increased degradation/efflux of the polyamines. In addition, the active transport of extracellular polyamines into the cell associates with cellular polyamine homoeostasis. In cytoplasm, the rate-limiting enzyme, SSAT acetylates the spermidine and spermine using acetyl-coenzyme A (CoA) to form N-acetylspermidine and $\mathrm{N}$ acetylspermine. Then acetylated forms of polyamines are oxidized by PAO and converted into putrescine and spermidine, respectively. ${ }^{3,4}$ SSAT is a key metabolic regulator in polyamine homeostasis. ${ }^{5}$ The overexpression of polyamineacetylating enzyme SSAT significantly increases biosynthesis and metabolic flux through the polyamine pathway. ${ }^{6}$ Via acetylation, the net positive charge of the polyamines declines and increased anionic character of polyamines alters the binding activity with biological molecules. ${ }^{3}$ The acetyl derivatives can be removed from the cells by transport and catabolism. Since SSAT is sensitive to cellular environment it rapidly alters its activity. SSAT basal activity is very low but increases quickly when polyamines are in excess. ${ }^{7}$ The short half-life of SSAT (20 min.) is also important to allow the cell to rapidly change the enzyme and polyamine levels. ${ }^{8}$ Two decarboxylase enzymes (ODC and AdoMetDC), the cytosolic acetyl-CoA:spermidine/spermine N1-acetyltransferase and a polyamine transport system are required for the regulation of cellular polyamine metabolism.

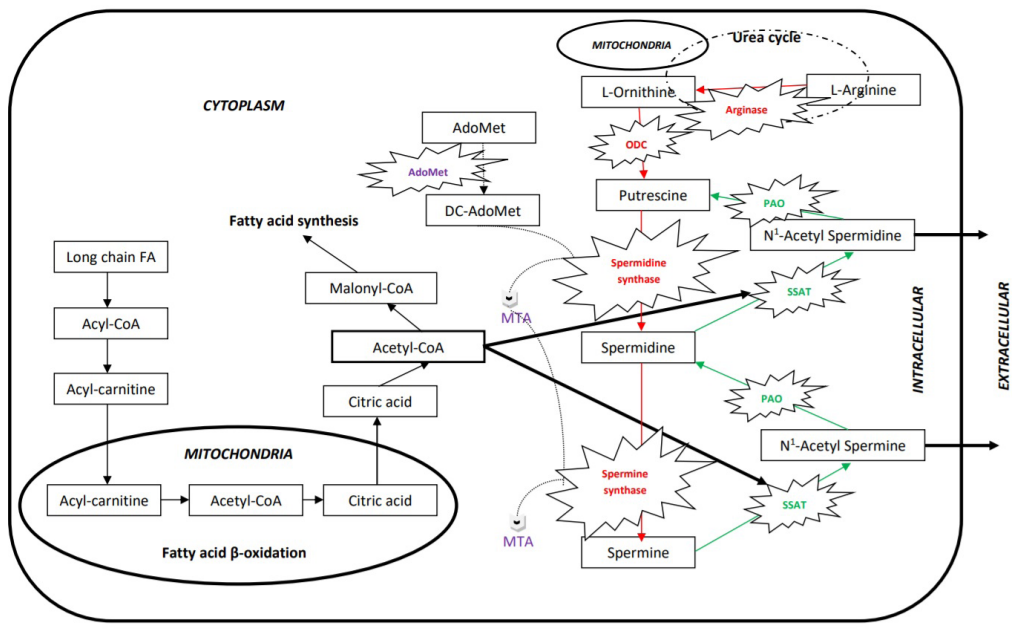

Figure 1. Metabolic pathways of polyamines and long chain fatty acids.

AdoMet: S-Adenosyl methionine, AdoMetDC: Adenosylmethionine decarboxylase, DC-AdoMet: Decarboxylated S-adenosylmethionine, MTA: 5'-methylthioadenosine, ODC: Ornithine decarboxylase, PAO: Polyamine oxidase, SSAT: Spermidine/spermine N-1 acetyl transferase. 
The levels of spermidine and spermine in cell are depended on polyamine homeostasis. Arginase catalysis the transformation of arginine to ornithine which is transformed to putrescine via ODC. Spermidine and spermine synthases catalyze the biosynthesis of spermidine from putrescine and spermine from spermidine respectively. The biosynthetic enzyme spermidine synthase uses DCAdoMet and putrescine to make spermidine and 5'-deoxy-5'-methylthioadenosine as a byproduct. The anabolic enzyme PAO deacetylates the N-Acetyl spermine and $\mathrm{N}$-Acetyl spermidine to keep polyamines in cell for required processes. Long chain FAs are oxidized to yield Acetyl-CoA in mitochondrial matrix via beta oxidation. Then, acetyl-CoA can be degraded to $\mathrm{CO}_{2}$ and $\mathrm{H}_{2} \mathrm{O}$ via the Krebs Cycle in mitochondria or used for fatty acid synthesis through Malonyl-CoA in cytoplasm. In other pathway, acetyl-CoA can be used for activation of SSAT which catalysis the formation of acetylated spermidine and spermine before taken out of the cell.

\section{Polyamine metabolism in obesity}

Obesity is a growing health problem worldwide and associated with many diseases such as type II diabetes, stroke, cardiovascular disease, and cancer..$^{9-11}$ In obesity, positive caloric intake causes excess fat stored in adipocytes which are increased by their size (hyperplasia) and number (hypertrophy). Increasing in fat tissue rise energy expenditure, so that higher energy intake is needed to maintain energy balance. Thus, body weight can be regulated by energy intake and energy expenditure.

Adipose tissue is an active endocrine organ and a main energy store of the body. Excess fat accumulation leads the release of free fatty acids into the circulation from adipocytes. The major energy reserve in higher eukaryotes is triacylglycerol in white adipose tissue (WAT). In case of energy deprivation or changes in nutritional state, lipolysis in adipocytes regulating through hormonal and biochemical signals proceeds.

Indeed, the rates of fuel utilization and fuel storage have been regulated by acetyland malonyl-CoA pools. In case of limited fuel, glycogen is broken down into glucose and glycolysis is up-regulated through the AMP-activated protein kinase (AMPK) activation. When the level of glucose increased, acetyl-CoA accumulation can quickly be remedied by increased polyamine acetylation. One cycle of polyamine flux utilizes two acetyl-CoA moieties. These moieties can be used for polyamine acetylation. Acetylated forms of polyamines are excreted into the urine thus acetyl-CoA cannot be used for storing excess energy as fat. Any lost in energy is noteworthy since 2 acetyl-CoA molecules have the equivalent of 24 ATP molecules. Moreover, increased polyamine acetylation decreases the availability of acetyl-CoA for malonyl-CoA synthesis and increases fatty acid oxidation. 
Excessive body weight associates with physiological mechanisms relating to control of fat deposition/mobilization. It has long been known that polyamines, specifically spermine and spermidine, stimulate adipose triacylglycerol formation by activation of several enzymes including sn-glycerol-3-phosphate acyltranferase, $\mathrm{Mg}^{2+}$-dependent phosphatidate phosphohydrolase and diacylglycerolacyl transferase. ${ }^{12}$ In addition, at physiological concentrations, both spermine and spermidine have been shown to inhibit lipolysis by suppressing cyclic AMP levels and to facilitate glucose transport, which is accompanied by up-regulated conversion of glucose into triacylglycerols in adipocytes of Zucker obese rats. ${ }^{13} \mathrm{~A}$ comparison of lean and obese Zucker rats showed rising concentration of spermine and spermidine which was accompanied by increasing in the activities of various triacylglycerol synthetic enzymes. ${ }^{14}$ The inhibition of ODC, the enzyme producing putrescine and a limiting step in polyamine biosynthesis, by an irreversible inhibitor alpha-difluoromethylornithine (DFMO) prevented the differentiation of $3 \mathrm{~T} 3-\mathrm{L} 1$ fibroblasts into adipocytes. In a recent paper, regulatory role of polyamines in adipogenesis was examined and it was shown that polyamines are required early in the adipogenic process. ${ }^{15}$

Polyamine flux is controlled by ODC, SSAT and PAO and has a role in energy metabolism. ${ }^{16,17}$ It was reported that perturbations of polyamine pathway flux were associated with obesity resistance or sensitivity in several mouse models. ${ }^{18,19}$ Nicotinamide N-methyltransferase (NNMT) methylates nicotinamide which is a precursor of $\mathrm{NAD}^{+}$using S-adenosylmethionine (SAM). It was shown that an increase in spermidine was also accompanied by reduced levels of NNMT which is associated with obesity resistance. ${ }^{18}$ Additionally, the increase in spermidine in $\mathrm{Maf1}^{-/-}$mice was accompanied by reduced levels of NNMT, which promotes polyamine synthesis, enables nicotinamide salvage to regenerate $\mathrm{NAD}^{+}$and is associated with obesity resistance. ${ }^{20}$ Therefore, it seems that targeting NNMT could be a promising approach for treating obesity and type 2 diabetes.

Regarding the proposed role of polyamines in hormone action, in a study by Grassi et al, it was suggested that polyamines may play a pivotal role in hormone responsiveness of hypothalamic-hypophyseal axis which was shown to contribute to the sympatho excitation of obesity. ${ }^{21}$ In obese and normal weight children luteinizing hormone ( $\mathrm{LH}$ ) values significantly increased after stimulation with acute gonadotropin-releasing hormone $(\mathrm{GnRH})$ injection but reached higher peaks in normal children than in obese ones. On the contrary, polyamine levels increased significantly only in the normal weight children. ${ }^{22}$

Genetic studies revealed a strong connection between SSAT activity and control of lipid metabolism. In transgenic mice, tissues were characterized by increased 
N1-acetyltransferase activity, a marked elevation in tissue and urinary acetylated polyamines, a compensatory increase in polyamine biosynthetic enzyme activity, and an increase in metabolic flux through the polyamine pathway. SSATdeficient mice developed insulin resistance at old age, possibly indicating that polyamine catabolism has a role in the regulation of glucose and energy metabolism. ${ }^{17}$ Male Gyro (Gy) mice with profound spermine deficiency due to the extensive disruption of both phosphate regulating endopeptidase and spermine synthase gene, exhibit the features of smaller size and lower weight than normal mice, whereas spermine synthase overexpressing mice are slightly larger than normal mice. ${ }^{23,24}$ In a study by Ishii et al, to explore the possible function of spermidine and spermine in adipogenesis, it was examined the effect of polyamine biosynthesis inhibitors, namely trans-4-methylcyclohexylamine (MCHA) and $\mathrm{N}$-(3-aminopropyl)-cyclohexylamine (APCHA), on adipocyte differentiation and lipid accumulation of $3 \mathrm{~T} 3-\mathrm{L} 1$ cells. They found that MCHA and APCHA have their own specific effects on both preadipocytes and mature adipocytes with the changes in SSAT activity suggesting that the control of polyamine metabolic enzyme activity could regulate adipogenesis. ${ }^{25}$ Moreover, there has been a close link between the disturbances of cellular metabolism and mitochondrial dysfunction. Mitochondrial abnormalities may cause to the development of common metabolic disorders such as type 2 diabetes and obesity. ${ }^{26}$ The activation of PGC-1a, the master regulator of mitochondrial biogenesis and energy expenditure, in the WAT and liver of mice resulted that WAT-specific SSAT over expression was sufficient to increase the number of mitochondria, reduce WAT mass and protect the mice from high-fat diet-induced obesity. ${ }^{27}$ Proteomics study on the liver tissues of SSAT-tg mice revealed changes in 23 proteins associated with polyamine catabolism..$^{28}$

In conclusion the polyamine metabolism enzymes modulate the acetyl-CoA flux associated with the lipolysis, energy metabolism and calorie loss influencing the accumulation of body fat. Any destruction on enzymes mainly SSAT, ODC, $\mathrm{PAO}$ and AdoMetDC reveals an alteration in the polyamine levels which may be associated with obesity. Therefore, in the future, polyamine metabolism should be considered as a valuable tool for research on lipid metabolism and drug development for obesity and related diseases. 


\section{CONFLICT OF INTEREST}

Authors declare that there is no conflict of interest.

\section{REFERENCES}

1. Pegg, A.E. Functions of polyamines in mammals. J. Biol. Chem. 2016, 291, 14904-14912.

2. Persson, L. Polyamine homoeostasis. Essays Biochem. 2009, 46, 11-24.

3. Wallace, H.M.; Fraser, A.V.; Hughes, A. A perspective of polyamine metabolism. Biochem J. 2003, 376, 1-14.

4. Perez-Leal, O.; Barrero, C.A.; Clarkson, A.B.; Merali, S. Polyamine-regulated translation of spermidine/spermine-N1-acetyltransferase. Mol. Cell Biol. 2012, 32(8), 1453-1467.

5. Pegg, A.E. Spermidine/spermine-N(1)-acetyltransferase: A key metabolic regulator. Am. J. Phsiol. Endocrinol. Metab. 2008, 294, E995-1010.

6. Kramer, D.L.; Diegelman, P.; Jell, J.; Vujcic, S.; Merali, S.; Porter, C.W. Polyamine acetylation modulates polyamine metabolic flux, a prelude to broader metabolic consequences. $J$. Biol. Chem. 2008, 283, 4241-4251.

7. Casero, R.A.; Pegg, A.E. Polyamine catabolism and disease. Biochem. J. 2oo9, 421, 323-338.

8. McCloskey, D.E.; Pegg, A.E. Properties of the spermidine/spermine N1-acetyltransferase mutant L156F that decreases cellular sensitivity to the polyamine analogue N1,N11-bis(ethyl) norspermine. J. Biol. Chem. 2003, 278, 13881-13887.

9. Pearson-Stuttard, J.; Zhou, B.; Kontis, V.; Bentham, J.; Gunter, M.J.; Ezzati, M. Worldwide burden of cancer attributable to diabetes and high body-mass index: a comparative risk assessment. Lancet Diabetes Endocrinol. 2018, 6(2), 95-104.

10. Agha, M.; Agha, R. The rising prevalence of obesity: part A: Impact on public health. Int. J. Surg. Oncol. 2017, 2(7):e17.

11. Bhupathiraju, S.N.; Hu, F.B. Obesity, diabetes, and cardiovascular diseases Compendium. Epidemiology of obesity and diabetes and their cardiovascular complications. Circ. Res. 2016, 118, 1723-1735.

12. Jamdar, S.C.; Osborne, L.J. Glycerolipid biosynthesis in rat adipose tissue: II. Effects of polyamines on $\mathrm{Mg}^{2+}$-dependent phosphatidate phosphohydrolase. Biochim. Biophys. Acta. 1983, 752, 79-88.

13. Lockwood, D.H.; East, L.E. Studies of the insulin-like actions of polyamines on lipid and glucose metabolism in adipose tissue cells. J. Biol. Chem. 1974, 249, 7717-7722.

14. Jamdar, S.C.; Cao, W.F.; Samaniego, E. Relationship between adipose polyamine concentrations and triacylglycerol synthetic enzymes in lean and obese Zucker rats. Enzyme Protein. 1996, 49, 222-230.

15. Brenner, S.; Bercovich, Z.; Feiler, Y.; Keshet, R.; Kahana, C. Dual regulatory role of polyamines in adipogenesis. J. Biol. Chem. 2015, 290, 27384-27392.

16. Jell, J.; Merali, S.; Hensen, M.L.; Mazurchuk, R.; Spernyak, J.A.; Diegelman, P. et al., Genetically altered expression of spermidine/spermine N1-acetyltransferase affects fat metabolism in mice via acetyl-CoA. J. Biol. Chem. 2007, 282, 8404-8413. 
17. Pirinen, E.; Kuulasmaa, T.; Pietila, M.; Heikkinen, S.; Tusa, M.; Itkonen, P. et al., Enhanced polyamine catabolism alters homeostatic control of white adipose tissue mass, energy expenditure, and glucose metabolism. Mol. Cell Biol. 2007, 27(13), 4953-4967.

18. Kraus, D.; Yang, Q.; Kong, D.; Banks, A.S.; Zhang, L.; Rodgers, J.T. et al., Nicotinamide Nmethyltransferase knockdown protects against diet-induced obesity. Nature. 2014, 508, 258-262.

19. Liu, C.; Leal, O.P.; Barrero, C.; Zahedi, K.; Soleimani, M.; Porter, C.; et al., Modulation of polyamine metabolic flux in adipose tissue alters the accumulation of body fat by affecting glucose homeostasis. Amino Acids. 2014, 46, 701-715.

20. Bonhoure, N.; Brynes, A.; Moir, R.D.; Hodroj, W.; Preitner, F.; Praz, V. et al., Loss of the RNA polymerase III repressor MAF1 confers obesity resistance. Genes Dev. 2015, 29, 934-947.

21. Grassi, G.; Seravalle, G.; Dell'Oro, R.; Turri, C.; Pasqualinotto, L.; Colombo, M. et al., Participation of thehypothalamus-hypophysisaxis inthe sympathetic activation of human obesity. Hypertension. 2001, 38, 1316-1320.

22. Bernasconi, S.; Orlandini, G.; Reali, N.; Guarneri, G.; Soldi, M.E.; Bacciottini, F. et al., Effect of GnRH administration on blood polyamines and LH levels in normal and obese children. Horm. Metab. Res. 1988, 20, 648-651.

23. Meyer, R.A.; Henley, C.M.; Meyer, M.H.; Morgan, P.L.; McDonald, A.G.; Mills, C. et al., Partial deletion of both the spermine synthase gene and the Pex gene in the X-linked hypophosphatemic, gyro (Gy) mouse. Genomics. 1998, 48, 289-295.

24. Wang, X.; Ikeguchi, Y.; McCloskey, D.E.; Nelson, P.; Pegg, A.E. Spermine synthesis is required for normal viability, growth, and fertility in the mouse. J. Biol. Chem. 2004, 279, 51370-51375.

25. Ishii, I.; Ikeguchi, Y.; Mano, H.; Wada, M.; Pegg, A.E.; Shirahata, A. Polyamine metabolism is involved in adipogenesis of 3T3-L1 cells. Amino Acids. 2012, 42, 619-626.

26. Auwerx, J. Improving metabolisms by increasing energy expenditure. Nat. Med. 2006, 12, 44-45.

27. Koponen, T.; Cerrada-Gimenez, M.; Pirinen, E.; Hohtola, E.; Paananen, J.; Vuohelainen, S. et al., The activation of hepatic and muscle polyamine catabolism improves glucose homeostasis. Amino Acids. 2012, 42(2-3), 427-440.

28. Cerrada-Gimenez, M.; Hayrinen, J.; Juutinen, S.; Reponen, T.; Janne, J.; Alhonen, L. Proteomic analysis of livers from a transgenic mouse line with activated polyamine catabolism. Amino Acids. 2010, 38, 613-622. 
